Article

\title{
Study of a QCM Dimethyl Methylphosphonate Sensor Based on a ZnO-Modified Nanowire-Structured Manganese Dioxide Film
}

\author{
Zhifu Pei ${ }^{1}$, Xingfa Ma ${ }^{2,3}$, Pengfei Ding ${ }^{1}$, Wuming Zhang ${ }^{1, *}$, Zhiyuan Luo ${ }^{4}$ and Guang Li ${ }^{1}$ \\ 1 National Key Laboratory of Industrial Control Technology, Department of Control Science and \\ Engineering, Zhejiang University, Hangzhou 310027, China; \\ E-Mails: florapzf@yahoo.com.cn(Z.P); dpfzjuv@126.com(P.D.); guangli@zju.edu.cn (G.L.) \\ 2 State Key Laboratory of Silicon Materials, Zhejiang University, Hangzhou 310027, China; \\ E-Mail: xingfamazju@yahoo.com.cn (X.M.) \\ 3 School of Environmental and Material Engineering, Center of Advanced Functional Materials, \\ Yantai University, Yantai 264005, China \\ 4 Computer Learning Research Centre, Royal Holloway, University of London, Egham, Surrey \\ TW20 0EX, UK; E-Mail: Zhiyuan.Luo@cs.rhul.ac.uk (Z.L.)
}

* Author to whom correspondence should be addressed; E-Mail: zhangwm@zju.edu.cn; Tel.: +86-571-8795-2268 ext. 2230; Fax: +86-571-8795-2279.

Received: 10 August 2010; in revised form: 20 August 2010 / Accepted: 31 August 2010 / Published: 2 September 2010

\begin{abstract}
Sensitive, selective and fast detection of chemical warfare agents is necessary for anti-terrorism purposes. In our search for functional materials sensitive to dimethyl methylphosphonate (DMMP), a simulant of sarin and other toxic organophosphorus compounds, we found that zinc oxide $(\mathrm{ZnO})$ modification potentially enhances the absorption of DMMP on a manganese dioxide $\left(\mathrm{MnO}_{2}\right)$ surface. The adsorption behavior of DMMP was evaluated through the detection of tiny organophosphonate compounds with quartz crystal microbalance (QCM) sensors coated with $\mathrm{ZnO}$-modified $\mathrm{MnO}_{2}$ nanofibers and pure $\mathrm{MnO}_{2}$ nanofibers. Experimental results indicated that the QCM sensor coated with $\mathrm{ZnO}$-modified nanostructured $\mathrm{MnO}_{2}$ film exhibited much higher sensitivity and better selectivity in comparison with the one coated with pure $\mathrm{MnO}_{2}$ nanofiber film. Therefore, the DMMP sensor developed with this composite nanostructured material should possess excellent selectivity and reasonable sensitivity towards the tiny gaseous DMMP species.
\end{abstract}


Keywords: quartz crystal microbalance; gas sensor; volatile organic vapor; DMMP; nanowire; manganese dioxide; zinc oxide

\section{Introduction}

Sensitive and selective detection of a wide variety of chemical species has become a necessity in many applications, including the quantification of chemical warfare agents (CWAs), explosives, environmental pollutants and many other toxic industrial compounds [1,2]. The threat of terrorism has greatly increased the need for fast detection of CWAs, so it is urgent to develop CWA sensors with fast response, high specificity, low detection limits and easy operation [3,4]. Dimethyl methylphosphonate [DMMP, $\mathrm{CH}_{3} \mathrm{PO}\left(\mathrm{OCH}_{3}\right)_{2}$ ] due to its nontoxicity and organophosphorus compound elemental composition that mimics nerve agents, is commonly considered as a simulant for CWAs and insecticides, such as the G-series nerve agents tabun (GA), sarin (GB), soman (GD) and paraoxon [5]. DMMP has also become a significant environmental and food chain pollutant due to its large consumption as a common additive for anti-foaming agents, plasticizers, stabilizers, textile conditioners and antistatic agents [2]. Consequently, DMMP sensors with high sensitivity, rapid response, low energy consumption and good reversibility at room temperature are highly desirable, not only for neurotoxin detection for counter-terrorism purposes, but also for environmental protection and medical diagnoses for risk management [6-8].

Focusing on highly sensitive functional materials with advanced fabrication technology, metal oxide semiconductors [8-11], carbon nanotubes [12-14], conducting polymers [15] and organometallic compounds [16,17] have been studied. Of these, metal oxides are well-known for their industrial applications as adsorbents, catalysts and catalyst supports, especially manganese dioxide $\left(\mathrm{MnO}_{2}\right)$ and titanium dioxide $\left(\mathrm{TiO}_{2}\right)$ which are widely used as molecular sieves and electrode materials in batteries and sensors due to their unique electronic and surface properties [18-22]. Furthermore, they are often used as sensing materials for different gases now due to their large gases adsorbent capacity $[8,23,24]$. Several investigations have been carried out concerning the adsorption and reaction of DMMP or other CWAs on the surfaces of different metal oxides, including $\mathrm{MgO}$ [25,26], $\mathrm{Al}_{2} \mathrm{O}_{3}$ [26-28], $\mathrm{TiO}_{2}$ [26,29-31], $\mathrm{Fe}_{2} \mathrm{O}_{3}$ [32,33], $\mathrm{ZnO}$ [26,34,35], $\mathrm{SiO}_{2}$ [36], $\mathrm{MnO}_{2}$ [37] and $\mathrm{WO}_{3}$ [26,38]. Zinc oxide $(\mathrm{ZnO})$ in particular shows very strong sensitivity toward toxic substances, such as halogens, sulfur, volatile organic compounds [35] and organophosphorus compounds [34,35]. Furthermore, the experiments on powdered $\mathrm{TiO}_{2}$ have revealed three distinct modes of adsorption: DMMP condenses on the outer surface of $\mathrm{TiO}_{2}$ below $160 \mathrm{~K}$; molecularly diffuses into the $\mathrm{TiO}_{2}$ interior and chemisorbs on $\mathrm{TiO}_{2}$ from 160 to $200 \mathrm{~K}$; and dissociatively chemisorbs above $214 \mathrm{~K}$ [30]. Enhancement to the absorption and reactivity of their nanoparticles and other nanostructures to sensitively detect various pollutants and harmful substances, including organophosphorus compounds, is anticipated due to their unique electronic properties, morphological features and high surface area [39]. These details indicate the nanostructured metal oxides like $\mathrm{TiO}_{2}, \mathrm{ZnO}$ and $\mathrm{MnO}_{2}$ may show sensitive and dissociative adsorbent of gas-phase DMMP at ambient temperature, promising sensing behavior for the mass detection of gas-phase organophosphorus compounds. However, only a few experimental studies have 
been reported on the adsorption of toxic chemicals or chemical warfare agent simulants on nano-structured $\mathrm{MnO}_{2}$ at ambient temperature [40].

In addition, it is interesting that the behavior of alumina-supported iron oxide may be significantly different from that of pure alumina [32,33]. Furthermore, zinc oxide doped in $\mathrm{SnO}_{2}$ may improve the reliability and sensitivity of the $\mathrm{SnO}_{2}$ sensors for simulants of the CWAs at 250 to $400{ }^{\circ} \mathrm{C}$ [40]. It is suggested that the absorption capability of $\mathrm{DMMP}$ on $\mathrm{MnO}_{2}$ surface is possibly improved by $\mathrm{ZnO}$ modification. In this study, we attempted to modify the $\mathrm{MnO}_{2}$ nanostructure with $\mathrm{ZnO}$ to explore new sensing materials and furthermore to evaluate the DMMP absorption properties at ambient temperature by constructing a sensitive DMMP sensor based on the $\mathrm{MnO}_{2}$ nanostructured film and the quartz crystal microbalance (QCM). Consequently, the adsorption behavior of DMMP on the composite material was characterized through the detection of tiny organophosphonate compounds with QCM sensors coated with $\mathrm{ZnO}$-modified $\mathrm{MnO}_{2}$ nanofibers and the comparison of these properties to those of the same sensors coated with pure $\mathrm{MnO}_{2}$ nanofibers. We thus concluded that these features make the developed DMMP sensor possess excellent selectivity and reasonable sensitivity.

\section{Experimental Section}

\subsection{Materials}

Chemicals and regents for nanostructured metal oxides were manganese (II) sulfate $\left(\mathrm{MnSO}_{4}\right)$, potassium permanganate $\left(\mathrm{KMnO}_{4}\right)$, zinc nitrate $\left[\mathrm{Zn}\left(\mathrm{NO}_{3}\right)_{2}\right]$, hexamethylenetetramine and ammonia. These chemicals and reagents were analytical grade and commercially available. The chemicals for volatile organic vapors (VOCs) were dimethyl methylphosphonate (DMMP), acetone, $p$-dichlorobenzene ( $p$-DCB), $p$-dimethylbenzene ( $p$-xylene), ethanol, $n$-hexane and trichloro-methane (chloroform) purchased from Sigma-Aldrich (Shanghai, China) and Wako Pure Chemicals (Osaka, Japan). All these chemicals and reagents were used directly as received without further purification. The AT-cut $6.0 \mathrm{MHz}(\mathrm{HC}-49 / \mathrm{U})$ quartz crystals with aluminum electrodes on both sides were purchased from Hosonic International (Hangzhou) Ltd., China. The crystals were rinsed by ethanol and then deionized water prior to use. All experiments were carried out at room temperature (about 25 degrees Celsius in an air-conditioned room).

\subsection{Preparation of pure and $\mathrm{ZnO}$-modified $\mathrm{MnO}_{2} \mathrm{NW}$-structured films}

\subsubsection{Preparation of NW-structured $\mathrm{MnO}_{2}$}

In the preparation process, $1.0 \mathrm{~g} \mathrm{MnSO}_{4}$ and $0.5 \mathrm{~g}$ of oxidizing reagent $\left(\mathrm{KMnO}_{4}\right)$ were dissolved in $20 \mathrm{~mL}$ of distilled water at room temperature to form a homogeneous solution. The solution was then transferred into a $100 \mathrm{~mL}$ Teflon-lined stainless steel autoclave, sealed and maintained at $120{ }^{\circ} \mathrm{C}$ for about 24 hours. After the resulting solid product was filtered and washed with distilled water to remove the possibly remnant ions in the final products and finally dried in air, $\mathrm{MnO}_{2} \mathrm{NWs}$, the final product was obtained. 


\subsubsection{Modification of $\mathrm{NW}$-structured $\mathrm{MnO}_{2}$ with $\mathrm{ZnO}$}

About $0.5 \mathrm{~g}$ of home-made $\mathrm{MnO}_{2} \mathrm{NWs}$ was dispersed in $20 \mathrm{~mL}$ distilled water; sequentially, $0.5 \mathrm{~g}$ zinc nitrate and $0.5 \mathrm{~g}$ hexamethylenetetramine were added. The mixture was then transferred into a Teflon-lined stainless steel autoclave. The $\mathrm{pH}$ value of reaction solution was adjusted to around 10 with ammonia. The hydrothermal treatments were carried out at $90-95{ }^{\circ} \mathrm{C}$ for 5 hours. After the resulting solid product was filtered, washed with distilled water repeatedly, and finally dried in air, the $\mathrm{ZnO}$-modified $\mathrm{MnO}_{2}$ nanowires (NWs) were obtained.

\subsubsection{Preparation of QCM sensors with $\mathrm{ZnO}$-modified $\mathrm{MnO}_{2} \mathrm{NW}$-structured film}

About $1 \mathrm{mg}$ of $\mathrm{ZnO}$-modified $\mathrm{NW}$-structured $\mathrm{MnO}_{2}$ was weighed and dispersed in deionized water to form a dark brown colored stock solution with a concentration of $2 \mu \mathrm{g} / \mu \mathrm{L}$. After standing for 24 hours, $2.5,5,7.5,10,12.5$ or 15 microliters of the aqueous solution was dispensed onto the electrode surface of QCMs using a micropipette, forming a sensing film with an area of $0.2 \mathrm{~cm}^{2}$ and a thickness index of $25,50,75,100,125$ or $150 \mu \mathrm{g} / \mathrm{cm}^{2}$, respectively. Then the device was dried in a dry cabinet at room temperature. After these steps, the QCM sensors coated with ZnO-modified $\mathrm{NW}$-structured $\mathrm{MnO}_{2}$ film were obtained.

\subsubsection{Structural characterization of the pure and $\mathrm{ZnO}$-modified $\mathrm{MnO}_{2}$ films on QCMs}

The nanostructure of $\mathrm{MnO}_{2}$ films were characterized by scanning electron microscopy (SEM). This observation was performed using a Field-Emission Scanning Electron Microscope with Energy Dispersive Spectrometer (FESEM-EDS, HITACHI S4800, Japan), operated at $25.0 \mathrm{kV}$. Both the pure and $\mathrm{ZnO}$-modified $\mathrm{MnO}_{2}$ films on the QCM sensors were deposited by platinum on the surface for SEM observation. The NW-structures of $\mathrm{MnO}_{2}$ films were observed and recorded.

\subsection{Experimental procedure for gas sensing}

The Sauerbrey equation was developed for oscillation in air and only applies to rigid mass attached to the crystal [41]. It gives the change in the oscillation frequency of piezoelectric quartz $(\Delta f)$ as a function of the mass $(\Delta \mathrm{m})$ added to the crystal:

$$
\Delta f=-\frac{2 f_{0}^{2}}{A \rho_{q} v_{q}} \Delta m
$$

Here, $\Delta f$ is the observed frequency change $(\mathrm{Hz}), f_{0}$ is the fundamental resonant frequency of crystal, $\mathrm{A}$ is the active area, the area where the crystal is coated with electrodes on both sides, $\rho_{\mathrm{q}}$ is the density of quartz and $v_{\mathrm{q}}$ is the shear wave velocity in the quartz. A home-made experimental system was set up to evaluate the as fabricated QCM sensors. The gas sensors set in the $500 \mathrm{~mL}$ sealed chamber of experimental setup were thus characterized at around $25{ }^{\circ} \mathrm{C}$ with either analytic gases for measurements or high-purity nitrogen gas for cleaning. The sensing-film-coated QCM was used as sensing unit while an uncoated QCM was used in the experimental system as reference. The variation of the frequency difference between the reference and sensing QCMs was defined as the response of QCM gas sensors. When the QCM DMMP sensor was exposed to the analyte DMMP, the sensing film 
would absorb the analytic gas, therein inducing a decrease in the working resonant frequency of the QCM DMMP sensors and the frequency change increase from a value at the start of the experiment. The working frequency of the QCM DMMP sensor in experimental setup changed from the fundamental resonant frequency of crystal coated with sensing film to a lower steady frequency of crystal decided by the sensing film-absorbed target gas. In this way, as the adsorption process approached equilibrium between the adsorption/desorption at a given concentration of DMMP, the frequency change or the response of QCM sensor increased, and finally reached a plateau phase. According to Equation (1) and adsorption mechanism [30], the steady value of frequency change at the plateau phase would determine the amount of gas absorbed in the film at ambient temperature. The absorbed DMMP analyte could be desorbed by high-purity $\mathrm{N}_{2}$, due to its dissociative adsorption at ambient temperature. The sensing QCM would thus be recovered; consequently the frequency change of the sensing QCM would be zeroed.

DMMP, acetone, $p$-DCB, $p$-xylene, ethanol, $n$-hexane and chloroform vapors were used as the analytical gases for this investigation. The experiments were performed as follows: first, a target analyte was injected into the testing chamber, the sensing film then absorbed the analyte, thus decreasing the output frequency of the sensing QCM. The response of the QCM gas sensor, or an increase of the frequency difference between the sensing and reference QCMs to the analyte, was measured continuously at a 1 -second interval $[42,43]$. After the response reached a plateau phase, the measurement course in a cycle was finished. The chamber was then purged with high-purity nitrogen gas to expel the analyte and recover the sensing films of sensors; the cleaning course for next cycle of measurement was started. This process was repeated several times for each analyte to get reliable results.

\section{Results and Discussion}

\subsection{Morphology of the sensing films}

Both the pure and $\mathrm{ZnO}$-modified $\mathrm{MnO}_{2}$ films on QCM sensors were investigated. The composite nanostructures in the $\mathrm{ZnO}$-modified $\mathrm{MnO}_{2}$ film can be seen in Figure 1.

Figure 1. SEM image of the $\mathrm{ZnO}$-modified $\mathrm{MnO}_{2}$ nanowire.

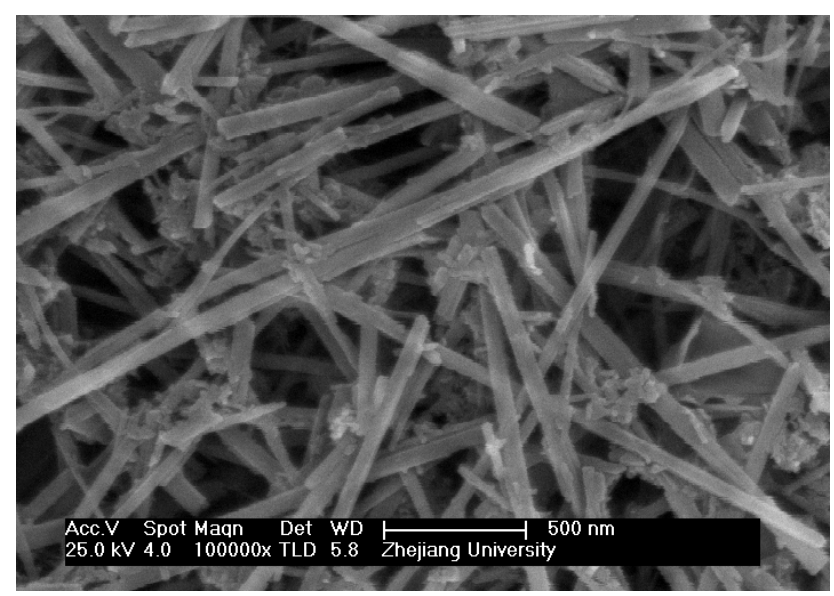

For comparison, the morphology and structure of the pure $\mathrm{NW}$-structured $\mathrm{MnO}_{2}$ film is shown in Figure 2. The observed data indicate that the $\mathrm{ZnO}$-nanoparticles modified the surface of $\mathrm{MnO}_{2} \mathrm{NWs}$. 
These SEM images confirmed that the composite nanostructures were formed in the ZnO-modified $\mathrm{MnO}_{2}$ film by the $\mathrm{ZnO}$ nanoparticles' joining to the $\mathrm{MnO}_{2} \mathrm{NWs}$. Therefore, we attribute the distinct sensing properties of the ZnO-modified $\mathrm{MnO}_{2} \mathrm{NW}$-structured QCM sensor to the composite nanostructures of the $\mathrm{ZnO}$ nanoparticle-modified surface of the $\mathrm{MnO}_{2} \mathrm{NWs}$.

Figure 2. SEM image of the pure $\mathrm{MnO}_{2}$ nanowires.

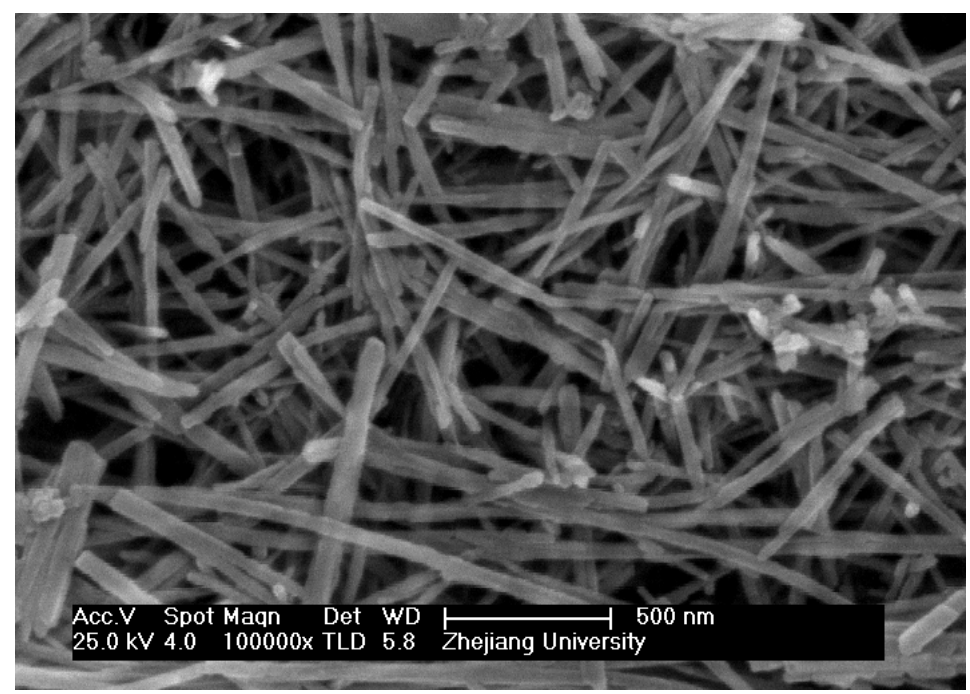

\subsection{Sensitivity and repeatability of the QCM DMMP sensors}

The QCM sensors based on ZnO-modified NW-structured $\mathrm{MnO}_{2}$ films were repeatedly tested at predefined DMMP concentrations for assessing their sensitivity and repeatability. At first, the QCM sensors coated with $\mathrm{ZnO}$-modified nanostructured $\mathrm{MnO}_{2}$ films were exposed to a series of defined concentrations of DMMP vapors diluted in high-purity nitrogen gas to assess their sensitivity. This investigation was performed by alternatively exposing the sensors to 300 -second DMMP vapors and 400-second nitrogen gas. The responses to the alternating inputs between the DMMP vapors of $0.35,0.70$ or $1.75 \mathrm{ppm}$ and cleaning gas of high purity nitrogen gas at room temperature were recorded and displayed in Figure 3.

Figure 3. Response cycles of a QCM sensor coated with ZnO-modified NW-structured $\mathrm{MnO}_{2}$ film towards $0.35,0.70$ and 1.75 ppms DMMP purged by high-purity nitrogen gas at room temperature.

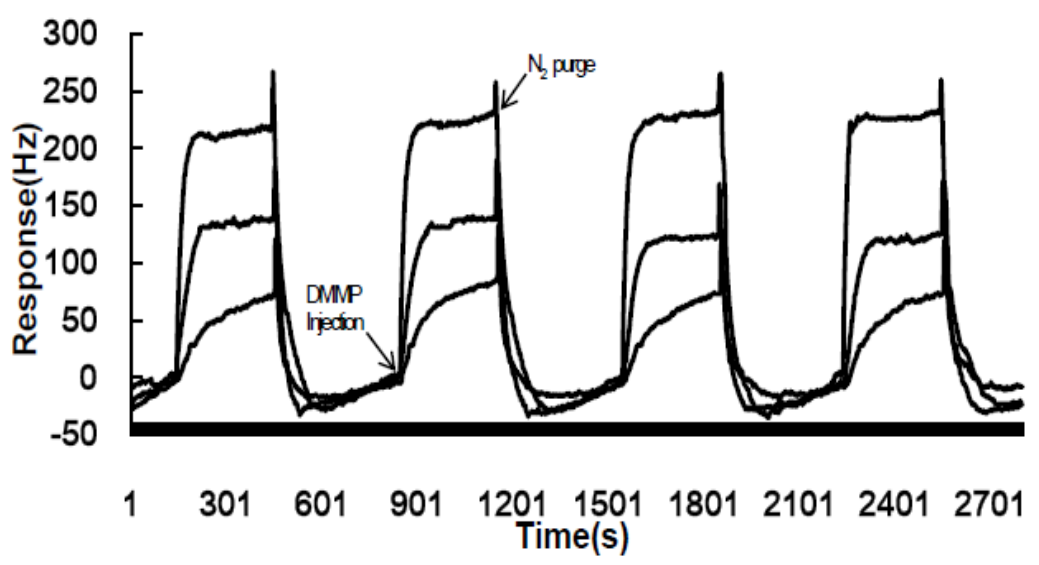


The response curves in Figure 3 indicate that the sensors were sensitive to the vapor concentrations and quickly responded to the high-purity nitrogen gas purge, thus possibly possessing a high sensitivity and good repeatability towards gaseous DMMP.

\subsection{Relationship between the responses (sensitivity) and the thickness of $\mathrm{ZnO}$-modified $\mathrm{MnO}_{2}$ NW-structured films}

The Sauerbrey equation generally gives a good prediction of the linear responses of the QCMs working in air, but only applies to rigid films [41]. The real situation of our developed QCM gas sensors to DMMP vapor sorption is much more complex, so there might be many challenges in predicting the linear relationship between the QCM sensor responses and the concentrations of DMMP vapors. Clearly, besides the nature and thickness of crystals (such as fundamental resonant frequency and $Q$ value), various factors related to the sensing films and target gases, such as the analyte-surface interaction and thickness of sensing films, can possibly contribute to the sensitivity and linearity of the sensor responses. For the QCM DMMP sensor based on $\mathrm{NW}$-structured $\mathrm{MnO}_{2}$ film, the film thickness is an important factor for suitable response when the material nature and fabrication method are given. The thickness of the sensing films on electrodes thus effects on both the mass-sensing properties of QCMs and physicochemical adsorption of DMMP.

To address this problem, we designed test experiments to investigate the relationship between the sensitivity of the sensors and the thickness of the sensing films on the sensors. Through these experiments, we could maximize the sensitivity and optimize the linearity of the QCM sensor by appropriately selecting the thickness of sensing films. The electrode surface on crystals was deposited with $2.5,5,7.5,10,12.5$ or 15 microliters of the $2 \mu \mathrm{g} / \mu \mathrm{L}$ aqueous solution of the $\mathrm{ZnO}$-modified $\mathrm{MnO}_{2}$ on $0.2 \mathrm{~cm}^{2}$ working area forming sensing films having six different thicknesses. Although the abstract values of these thicknesses could be not figured out due to lack of the mass density of the film, the thickness indices could be easily obtained by supposing that the mass density of film is constant for all films produced with same material and fabrication method. Thus, the $\mathrm{ZnO}$-modified $\mathrm{MnO}_{2}$ composite film deposited on sensors had a thickness index of $25,50,75,100,125$ or $150 \mu \mathrm{g} / \mathrm{cm}^{2}$, respectively. This thickness index reflects the variation of film thickness, thus defined and used as thickness in this study. The test experiments were performed during the produced QCM sensor was exposed in $0.7 \mathrm{ppm}$, a predefined concentration, of gaseous DMMP. The experimental findings, displayed in Figure 4, reveal a significant nonlinear relationship between the sensitivity of the sensors and the thickness of the sensing films.

Here, we tried to explain the nonlinear properties of the QCM sensor through the behaviors of QCM oscillation and DMMP adsorption. Both of them are closely related to the thickness of sensing film deposed on QCM substrate. The nonlinear phenomena seems partly contributed to the interference of the sensing film with the vibration state of QCM substrate. Nevertheless, the Sauerbrey equation still works on the QCM gas sensor. In principle, the responses of QCM sensor generally arise from both gravimetric and viscoelastic changes in real sensing films, whereas the Sauerbrey equation predicts the outputs according to the gravimetric changes in ideal rigid films. 
Figure 4. Responses of the QCM sensors with various thicknesses of sensing films to a predefined concentration of DMMP (0.7 ppm).

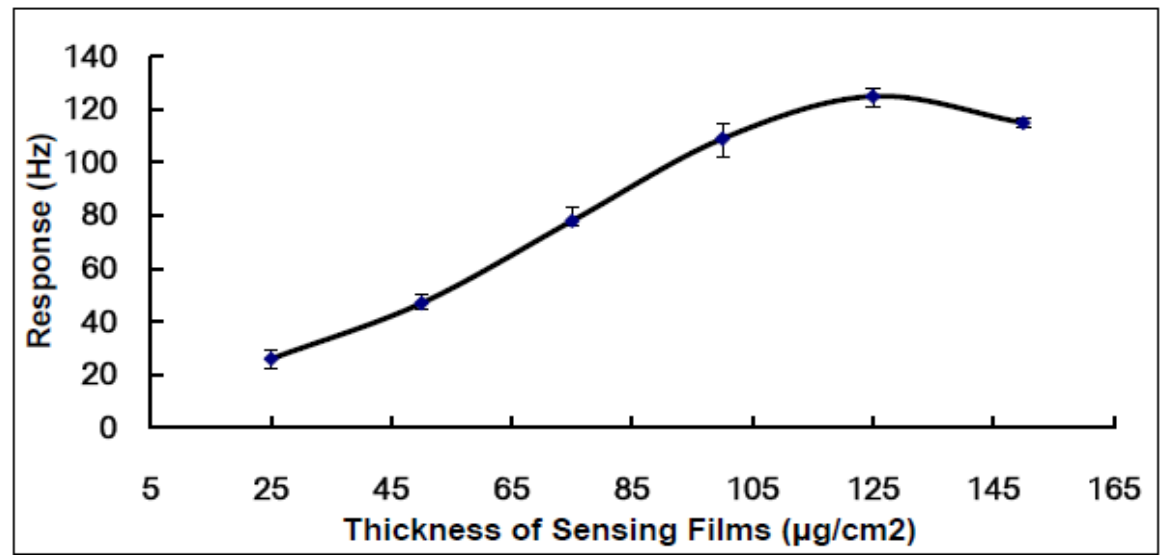

The sensing film moves synchronously with the underlying QCM substrate if the thickness of the coated sensing film is small enough relative to the acoustic wavelength in the film [45]. In this situation, the motion imparted by the QCM substrate displaces the sensing film parallel to the surface of the substrate just as the situation in a rigid film. The responses to the sensing film thus obey the Sauerbrey equation and reflect the gravimetric changes of the sensing film regardless of its shear modulus [46]. On the other hand, when the sensing thickness is big enough or acoustically thick (thicker than a few percents of the acoustic wavelength) the thickness stress gradients will become important. In this situation, the responses to the $\mathrm{NW}$-structured $\mathrm{MnO}_{2}$ sensing film will depend on both the acoustic thickness and the thickness shear modulus of the sensing film prior to and during DMMP vapor exposure [45-48], thus no longer accurately obeying the Sauerbrey equation. The experimental results, shown in Figure 4, reveal some of these effects. As is known, these nonlinear phenomena contribute to the variation of the QCM sensor's static working status consisting of the resonant frequency, Q value and other parameters prior to DMMP vapor exposure, as well as the dynamic sensing status of the film during DMMP vapor exposure, which will be described in the next paragraph. Despite working in a static working point different from that of theoretical crystal, the real QCM DMMP sensor can detect the mass changes of sensing film according to the Sauerbrey equation. That is why the QCM had been utilized in chemical and biological sensors in so many applications for so many years.

In our opinion, the nonlinear phenomena seem to contribute more significantly to the gas-surface interaction and adsorption of DMMP inside the $\mathrm{ZnO}$-modified $\mathrm{NW}$-structured $\mathrm{MnO}_{2}$ sensing film. The uniform and porosity structures of the NWs in film lead to a huge surface-to-volume ratios; these ratios, in turn, make the interaction between the sensing film and DMMP vapor efficient, thus, leading to quick and thorough equilibrium analyte absorption/desorption state. When the sensing film on the QCM substrate is thin, the whole sensing film, as an effective surface adsorption area, effectively adsorbs the target analyte. Thus, while the QCM sensor's responses reflect the gas diffusion and adsorption/desorption process in sensing film exposed in a fixed concentration of DMMP vapor, the QCM sensor's plateau responses reflect equilibrium status of adsorption/desorption to a given DMMP concentration. The readouts of the plateau curve are consequently the responses of the QCM sensor to a given DMMP concentration. The effective surface adsorption area will increase together with the 
amount, or the thickness of sensing materials deposited on a given area of substrate. Therein, the total amount of the DMMP absorbed, furthermore, the responses of the sensor exposed in a given DMMP concentration will also increase together with the thickness of sensing materials, as the linear line between 0.03 and $125 \mu \mathrm{g} / \mathrm{cm}^{2}$ shown in Figure 4. When the thickness is big, the DMMP molecule will penetrate a larger depth inside the sensing film to diffuse to the bottom of sensing film and reach equilibrium status of adsorption/desorption in whole sensing film. The responses of QCM sensor present more sensitive readouts to a defined DMMP concentration in spite of taking a longer time. However, when the amount is big enough, the sensing film is so thick that the DMMP molecules can only penetrate through the upper layer, and cannot reach to the bottom of the sensing film. Thus, the DMMP molecules will approach the equilibrium status of adsorption/desorption in the somewhat steady upper layer of the sensing film. Consequently, the effective surface adsorption area will no longer increase with the thickness of sensing films and the responses of the sensor to a given DMMP concentration tend to be stead too, shown as the plateau phase of the curve. The findings also illustrate that a sensing film with a thickness near to $125 \mu \mathrm{g} / \mathrm{cm}^{2}$ is most sensitive to $0.7 \mathrm{ppm}$ of DMMP vapor. We recognized this thickness value as the optimal thickness of the ZnO-modified NW-structured $\mathrm{MnO}_{2}$ film coated on QCM under the predefined experimental conditions. We consequently thought that the gas sensors set at this working situation would have a maximized sensitivity to $0.7 \mathrm{ppm}$, therein designing the sensors with this thickness of sensing films for the measurement range containing $0.7 \mathrm{ppm}$ and all other experiments in this study.

Anyway, how to find out the optimal thickness for designing QCM sensors with the best linearity and sensitivity to organic vapors is a challenge. As analyzed in the paragraphs above, despite many efforts, the gas diffusion and gas-surface interaction in sensing films are still far from being well understood for QCM metal oxide gas sensors. Further theoretical, empirical, or experimental results would be expected to understand how the gas concentration profile develops inside a thin film of metal oxide NWs after its exposure to a target gas, thus enabling us to design the sensing film more rationally.

\subsection{Relationship between the sensitivity and concentration of DMMP vapor}

For each designed sensor, its sensitivity was assessed at various concentrations of DMMP vapors. In order to evaluate the repeatability, three or four consecutive measurements at each concentration were required. Thus, the response $(\mathrm{R})$ to each given DMMP concentration $(\mathrm{C})$ was repeatedly measured three or four times. The results over a range from $0.035 \mathrm{ppm}$ to $2.8 \mathrm{ppm}$ were shown in Figure 5, where the small plot indicated the QCM sensor based on ZnO-modified NW-structured $\mathrm{MnO}_{2}$ film began to illustrate discernible nonlinearity at a DMMP concentration of near $3 \mathrm{ppm}$.

In contrast, as shown in the large plot in Figure 5, the responses of the sensor were almost linearly proportional to the lower DMMP concentrations, ranging from 0.035 to $1.05 \mathrm{ppm}$. The regression equation could be expressed as $\mathrm{R}=176.04 \mathrm{C}+3.01$ with a correlation coefficient of 0.9987 , where $\mathrm{C}$ is the concentration of DMMP vapors and $\mathrm{R}$ is the response or sensitivity of the sensor, respectively. This relationship plots the calibration curve of the $\mathrm{ZnO}$-modified $\mathrm{NW}$-structured $\mathrm{MnO}_{2}$ based QCM sensor. Accordingly, the limit of detection (calculated as three times the signal-to-noise ratio) could be estimated and given as $35 \mathrm{ppb}$. 
This result represents a great improvement compared to those previously published by other groups. Brunol et al. reported their study to deal with the DMMP detection, using tin dioxide-based gas sensors. They used a DMMP vapor concentration level of around $200 \mathrm{ppm}$ [6]. Ying et al. studied a PVDF coated QCM as the DMMP sensor [7]. The sensitivity was $3.19 \mathrm{~Hz} / \mathrm{ppm}$ over the range from 5 to $60 \mathrm{ppm}$ DMMP in $\mathrm{N}_{2}$ and the limit of detection was about $0.94 \mathrm{ppm}$. In comparison with their results, the sensor reported in this paper is highly sensitive, and thus suitable for low level DMMP detection.

Figure 5. Linear plot of the reciprocal of a $\mathrm{ZnO}$-modified nanowire $\mathrm{MnO}_{2}$ coated QCM sensor's response against the concentrations of DMMP vapor purged in high-purity nitrogen at room temperature.

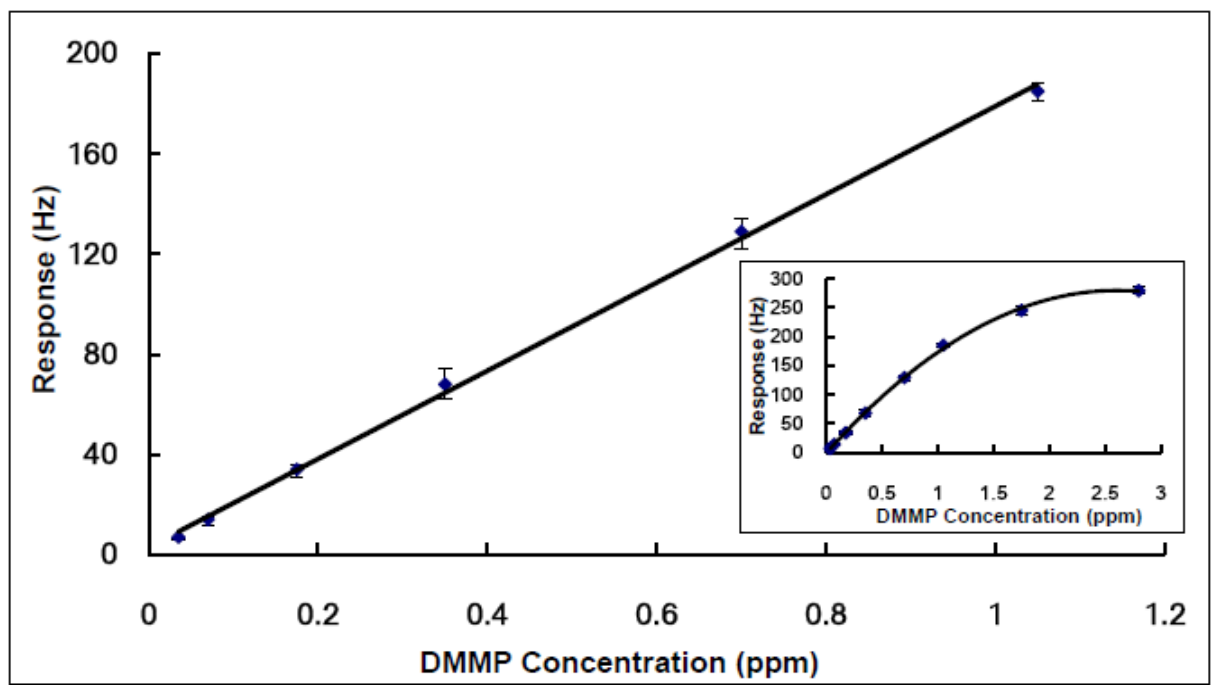

Although the frequency change of QCM responded linearly to the amount of gas absorbed and illustrated a high sensitivity to DMMP vapors, this sensor demonstrated nonlinearity at a large concentration range of DMMP, which was different from the description based on the Sauerbrey equation [41]. This nonlinearity might be contributed to by the thickness effects of the sensing film because the QCM works on a balanced status between the sensitivity and linearity. In principle, the thicker thickness of $\mathrm{ZnO}$-modified $\mathrm{NW}$-structured $\mathrm{MnO}_{2}$ film possesses much more effective surface adsorption area to adsorb DMMP; this makes the QCM sensor much more sensitive. However, this thicker thickness also leads to the static working status of the QCM sensor being different from that of the theoretical QCM, thus producing slight nonlinear effects on the Sauerbrey equation. More importantly, as described in Section 3.3, a thicker sensing film also induces nonlinear DMMP adsorption. These facts indicate that the QCM DMMP sensor shows more observable nonlinearity. Thus the thickness of the sensing film is one of the key factors affecting not only the sensitivity, but also the linearity of a sensor.

\subsection{Response of the sensor towards various organic vapors (selectivity)}

To investigate the selectivity, the QCM sensors were tested against several VOCs according to the instructions described above. The responses of the sensor exposed to a defined concentration of 0.7 ppm DMMP and potential interfering VOCs including acetone, chloroform, $p$-DCB, ethanol, 
$n$-hexane and $p$-xylene were measured. Each of these VOCs is usually used as solvents and may act as potential interferences. The amplitudes of the responses to the target gas-DMMP vapor as well as control vapors at same concentration of $0.7 \mathrm{ppm}$ were shown as in Figure 6. As we can see from the Figure, the response to the target DMMP vapor was much larger than those to the acetone, chloroform, $p$-DCB, ethanol, $n$-hexane and $p$-xylene vapors. Therefore, these findings indicated that the developed $\mathrm{ZnO}$-modified $\mathrm{MnO}_{2} \mathrm{NW}$-structured sensor possesses a very high selectivity to gaseous DMMP from VOCs including acetone, chloroform, $p$-DCB, ethanol, $n$-hexane and $p$-xylene.

Figure 6. Comparison of the responses of the sensor to various organic vapors diluted to a predefined concentration of $0.7 \mathrm{ppm}$.

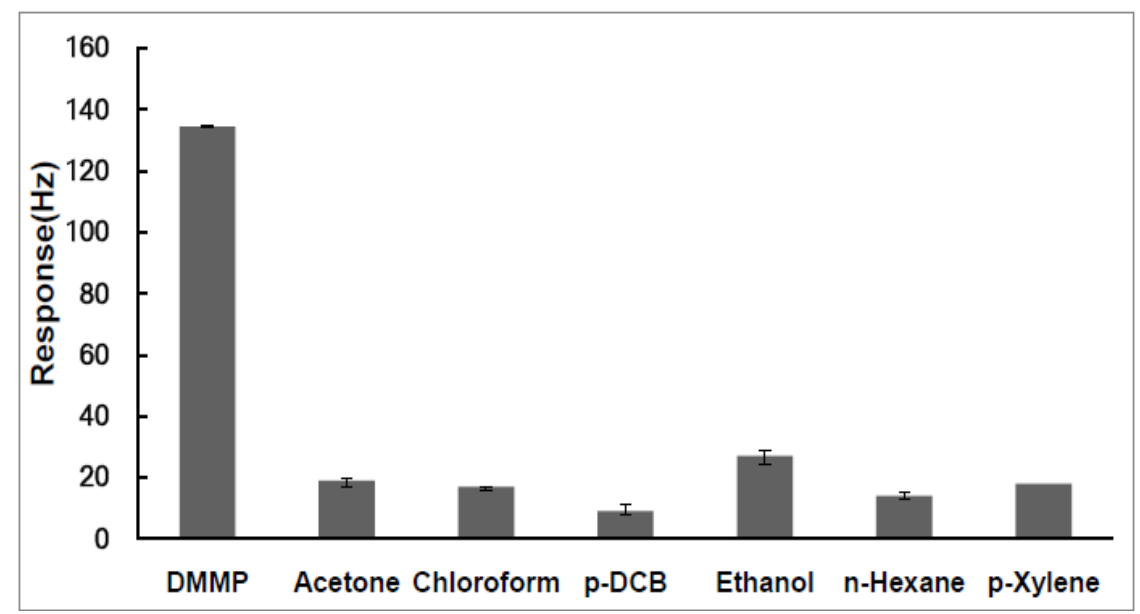

3.6. Comparison of the sensitivity between the sensors coated with $\mathrm{ZnO}$-modified $\mathrm{MnO}_{2}$ and pure $\mathrm{MnO}_{2}$

In order to assess the effect of the $\mathrm{ZnO}$-modified $\mathrm{MnO}_{2} \mathrm{NW}$-structured film on DMMP sensing, a reference QCM sensor with a same structure but pure $\mathrm{MnO}_{2} \mathrm{NW}$ film was fabricated for comparison. The responses of the reference QCM sensor to both the DMMP vapor and interfering VOCs including acetone, chloroform, $p$-DCB, ethanol, $n$-hexane and $p$-xylene at a same predefined concentration of $0.7 \mathrm{ppm}$ were investigated according to the instructions described above. The response amplitudes of these paired QCM sensors were compared and are shown in Figure 7. The response of $\mathrm{NW}$-structured pure $\mathrm{MnO}_{2}$-based QCM sensor to DMMP vapor was not higher than those to the potentially interfering VOCs; one example is that the sensitivity amplitude to the DMMP vapor was even slightly smaller than the one to chloroform. Clearly, these facts indicate that the QCM sensor based on NW-structured pure $\mathrm{MnO}_{2}$ did not present a usable selectivity to DMMP. In contrast, as shown in Figure 6, through the $\mathrm{ZnO}$ modification of $\mathrm{MnO}_{2} \mathrm{NWs}$, the sensitivity of the $\mathrm{MnO}_{2} \mathrm{NW}$ film based QCM sensor to the DMMP vapor was greatly increased although that to the potentially interfering VOCs was almost unchanged. We thus contributed this improvement of the selectivity to DMMP to the $\mathrm{ZnO}$ modification on $\mathrm{MnO}_{2} \mathrm{NWs}$, or the formed composite nanostructures shown as in Figure 1.

Understanding the interaction of phosphonate esters with the surfaces of $\mathrm{ZnO}$-modified $\mathrm{MnO}_{2} \mathrm{NWs}$ at room temperature is a challenge; this problem is critical for the development of sensors to measure CWAs. However, very little is known about mechanism of DMMP specific binding to metal oxides 
although a few researchers have studied the details of DMMP absorbance. In this study, the $\mathrm{MnO}_{2}$ nanowires-based gas sensor responded to the VOCs very well in comparison with most gas sensors, but as displayed in Figure 6, presenting an average to low selectivity. In this study, the nanocrystalline $\mathrm{ZnO}$ was utilized to modulate the strong catalytic activity of $\mathrm{MnO}_{2}$, forming heterogeneous interfaces for VOCs testing. The heterogeneous interfaces formed possibly reduce the catalysis of $\mathrm{MnO}_{2}$ to some VOC vapors, but increase that to other VOC vapors. These distinctive properties of this developed composite nanostructured material were evaluated through the QCM gas sensing. They displayed as the sensitivity and selectivity of QCM sensor to DMMP on device level.

Figure 7. Comparison of the responses between the sensor based on pure $\mathrm{MnO}_{2}$ nanowire film and $\mathrm{ZnO}$-modified $\mathrm{MnO}_{2}$ nanowire film to various organic vapor diluted to a predefined concentration of $0.7 \mathrm{ppm}$.

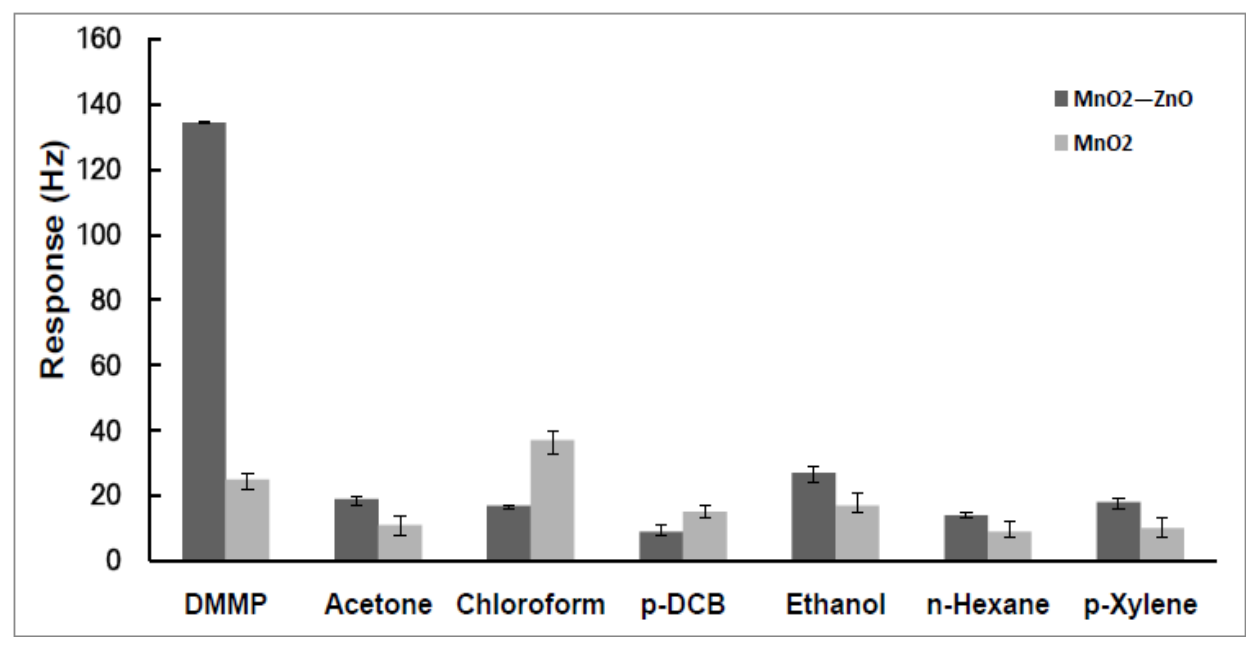

Interestingly, the experimental results indicated that the sensitivity and selectivity to the DMMP vapor was greatly increased whereas that to the VOC vapors was only slightly changed. We can thus contribute the improvement of the selectivity to the DMMP vapor to the modulation effects of the $\mathrm{ZnO}$ nanoparticles on the $\mathrm{MnO}_{2} \mathrm{NW}$-structured film. Of course, further study would be helpful to understand the detailed mechanisms at molecular level, and furthermore to improve the sensitivity and selectivity. Mitchell et al. have explored the uptake mechanisms of DMMP to the effective sorbent and reaction material $\mathrm{TiO}_{2}$ and considered that the DMMP molecule interacts through the electron-rich phosphoryl oxygen with surface-bound hydroxyl groups and with Lewis acid sites of the $\mathrm{TiO}_{2}$ [31]. Similar studies will be valuable to understand the mechanisms of DMMP effective absorption to the surface of the $\mathrm{ZnO}$-modified $\mathrm{MnO}_{2} \mathrm{NWs}$ at room temperature.

\subsection{Long-term stability}

In order to assess the long-term stability, the response of the sensor to a predefined concentration of the DMMP in nitrogen gas, that is, $0.54 \mathrm{ppm}$, the average concentration of the linear range from 0.035 to $1.05 \mathrm{ppm}$, had been regularly examined for 10 days as the instructions described. After each testing, the sensor was immediately stored in a dry cabinet at room temperature. The response changes over ten-day period are shown in Figure 8. The results shown in Figure 8 indicated that the response was relative stable and remained above $90 \%$ of its original value after 10 days. 
Figure 8. Responses of a nanowire $\mathrm{ZnO}_{2} / \mathrm{MnO}_{2}$ composite film based QCM sensor to a predefined concentration of 0.54 ppm DMMP in nitrogen gas during 10 days.

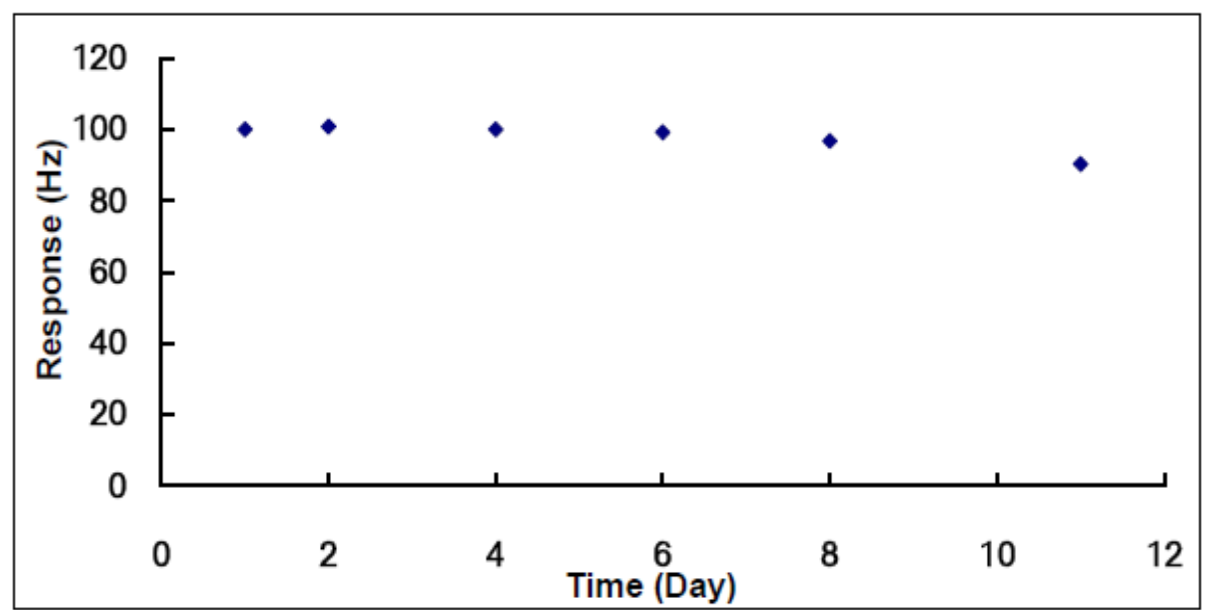

\section{Conclusions}

The preparation of highly active sensing film is believed to be a crucial step in obtaining sensitive and selective CWA sensors. For this purpose a composite nanostructured $\mathrm{MnO}_{2}$ material was produced in this study by the $\mathrm{ZnO}$ modification of $\mathrm{MnO}_{2}$ nanofibers. To evaluate its specific absorbing properties, both pure $\mathrm{MnO}_{2}$ and $\mathrm{ZnO}$-modified $\mathrm{MnO}_{2} \mathrm{NW}$-structured films were prepared on QCMs for DMMP sensing. With the $\mathrm{ZnO}$-modified device working at room temperature, a linear response to DMMP and a limit of detection of $35 \mathrm{ppb}$ were obtained, even though the DMMP was diluted to a concentration lower than $1.05 \mathrm{ppm}$. The data presented in this study thus show that the $\mathrm{ZnO}$ modification induces the $\mathrm{NW}$-structured $\mathrm{MnO}_{2}$ film effectively absorb DMMP vapor, thus improved significantly the sensitivity and selectivity of sensors. Therefore, this work provides valuable data with CWA simulant supporting the development of new nanostructured material as a sensitive film for the nerve agent and insecticide detection. The composite nanostructured DMMP-sensing film combined with the simple and low-cost QCM detection provides a promising configuration to develop practical chemical warfare gas sensor.

\section{Acknowledgements}

This research is supported by the Natural Science Foundation of China (Grants No. 60874098 and 60911130129), the National High Technology Research and Development Program of China (863 Program 2007AA042103) and the National Creative Research Groups Science Foundation of China (NCRGSFC: 60421002).

\section{References}

1. Munro, N.B.; Ambrose, K.R.; Watson, A.P. Toxicity of the organophosphate chemical warfare agents GA, GB, and VX: Implications for public protection. Envir. Health Persp. 1994, 102, 18-38.

2. Rudel, R.A.; Perovich, L.J. Endocrine disrupting chemicals in indoor and outdoor air. Atmos. Envir. 2009, 43, 170-181. 
3. Wils, E.R.J.; Hulst, A.G. The use of thermospray-liquid chromatography/mass spectrometry for the verification of chemical warfare agents. Fresenius J. Anal. Chem. 1992, 342, 749-758.

4. Otake, T.; Yoshinaga, J.; Yanagisawa, Y. Analysis of organic esters of plasticizer in indoor air by GC-MS and GC-FPD. Envir. Sci. Tech. 2001, 35, 3099-3102.

5. Bartelt-Hunt, S.L.; Knappe, D.R.U.; Barlaz, M.A. A review of chemical warfare agent simulants for the study of environmental behavior. Critical Rev. Envir. Sci. Tech. 2008, 38, 112-136.

6. Brunol, E.; Berger, F.; Fromm, M.; Planade, R. Detection of dimethyl methylphosphonate (DMMP) by tin dioxide-based gas sensor: Response curve and understanding of the reactional mechanism. Sens. Actuat. B 2006, 120, 35-41.

7. Ying, Z.; Jiang, Y.; Du, X.; Xie, G.; Yu, J.; Wang, H. PVDF coated quartz crystal microbalance sensor for DMMP vapor detection. Sens. Actuat. B 2007, 125, 167-172.

8. Comini, E.; Faglia, G.; Sberveglieri, G.; Pan, Z.W.; Wang, Z.L. Stable and highly sensitive gas sensors based on semiconducting oxide nanobelts. Appl. Phys. Lett. 2002, 81, 1869-1871.

9. Zhang, J.; Hu, J.Q.; Zhu, F.R.; Gong, H.; O'Shea, S.J. ITO thin films coated quartz crystal microbalance as gas sensor for NO detection. Sens. Actuat. B 2002, 87, 159-167.

10. Tomchenko, A.A.; Harmer, G.P.; Marquis, B.T.; Allen, J.W. Semiconducting metal oxide sensor array for the selective detection of combustion gases. Sens. Actuat. B 2003, 93, 126-134.

11. Lee, D.; Kim, Y.; Huh, J.; Lee, D. Fabrication and characteristics of $\mathrm{SnO}_{2}$ gas sensor array for volatile organic compounds recognition. Thin Solid Films 2002, 416, 271-278.

12. Chopra, S.; Pham, A. Carbon-nanotube-based resonant-circuit sensor for ammonia. Appl. Phys. Lett. 2002, 80, 4632-4634.

13. Modi, A.; Koratkar, N.; Lass, E.; Wei, B.; Ajayan, P. Miniaturized gas ionization sensors using carbon nanotubes. Nature 2003, 424, 171-174.

14. Kong, J.; Franklin, N.; Zhou, C.; Chapline, M.; Peng, S.; Cho, K.; Dai, H. Nanotube molecular wires as chemical sensors. Science 2000, 287, 622-625.

15. Matsuguchi, M.; Io, J.; Sugiyama, G.; Sakai, Y. Effect of $\mathrm{NH}_{3}$ gas on the electrical conductivity of polyaniline blend films. Syn. Metals 2002, 128, 15-19.

16. Miyata, T.; Kawaguchi, S.; Ishii, M.; Minami, T. High sensitivity chlorine gas sensors using Cu-phthalocyanine thin films. Thin Solid Films 2003, 425, 255-259.

17. Rakow, N.; Suslick, K. A colorimetric sensor array for odour visualization. Nature 2000, 406, 710-712.

18. Huang, H.; Mao, S.; Feick, H.; Yan, H.; Wu, Y.; Kind, H.; Weber, E.; Russo, R.; Yang, P. Room-temperature ultraviolet nanowire nanolasers. Science 2001, 292, 1897-1899.

19. Ammundsen, B.; Paulsen, J. Novel lithium-ion cathode materials based on layered manganese oxides. Adv. Mater. 2001, 13, 943-956.

20. Giraldo, O.; Brock, S.; Willis, W.; Marquez, M.; Suib, S.; Ching, S. Manganese oxide thin films with fast ion-exchange properties. J. Am. Chem. Soc. 2000, 122, 9330-9331.

21. Wang, X.; Li, Y.; Synthesis and formation mechanism of manganese dioxide nanowires/nanorods. Chem. Eur. J. 2003, 9, 300-306.

22. Perez-Lopez, O.W.; Farias, A.C.; Marcilio, N.R.; Bueno, J.M.C. The catalytic behavior of zinc oxide prepared from various precursors and by different methods. Mater. Res. Bull. 2005, 40, 2089-2099. 
23. Berna, A. Metal oxide sensors for electronic noses and their application to food analysis. Sensors 2010, 10, 3882-3910.

24. Kanan, S.M.; El-Kadri, O.M.; Abu-Yousef, I.A.; Kanan, M.C. Semiconducting metal oxide based sensors for selective gas pollutant detection. Sensors 2009, 9, 8158-8196.

25. Michalkova, A.; Ilchenko, M.; Gorb, L.; Leszczynski, J. Theoretical study of the adsorption and decomposition of sarin on magnesium oxide. J. Phys. Chem. B 2004, 108, 5294-5303.

26. Aurian-Blajeni, B.; Boucher, M.M. Interaction of dimethyl methylphosphonate with metal oxides. Langmuir 1989, 5, 170-174.

27. Bermudez, V.M. Quantum-chemical study of the adsorption of DMMP and sarin on $\gamma-\mathrm{Al}_{2} \mathrm{O}_{3}$. $J$. Phys. Chem. C 2007, 111, 3719-3728.

28. Bermudez, V.M. Computational study of environmental effects in the adsorption of DMMP, sarin, and $\mathrm{VX}$ on $\gamma-\mathrm{Al}_{2} \mathrm{O}_{3}$ : photolysis and surface hydroxylation. J. Phys. Chem. C 2009, 113, 1917-1930.

29. Trubitsyn, D.A.; Vorontsov, A.V. Experimental study of dimethyl methylphosphonate decomposition over anatase $\mathrm{TiO}_{2}$. J. Phys. Chem. B 2005, 109, 21884-21892.

30. Rusu, C.N.; Yates, J.T., Jr. Adsorption and decomposition of dimethyl methylphosphonate on $\mathrm{TiO}_{2}$. J. Phys. Chem. B 2000, 104, 12292-12298.

31. Mitchell, M.B.; Sheinker, V.N.; Mintz, E.A. Adsorption and decomposition of dimethyl methylphosphonate on metal oxides. J. Phys. Chem. B 1997, 101, 11192-11203.

32. Tesfai, T.M.; Sheinker, V.N.; Mitchell, M.B. Decomposition of dimethyl methylphosphonate (DMMP) on alumina-supported iron oxide. J. Phy. Chem. B 1998, 102, 7299-7302.

33. Mitchell, M.B.; Sheinker, V.N.; Cox, W.W., Jr. Room temperature reaction of ozone and dimethyl methylphosphonate (DMMP) on alumina-supported iron oxide. J. Phys. Chem. C 2007, 111, 9417-9426.

34. Paukku, Y.; Michalkova, A.; Leszczynski, J. Quantum-chemical comprehensive study of the organophosphorus compounds adsorption on zinc oxide surfaces. J. Phys. Chem. C 2009, 113, 1474-1485.

35. Oha, S.W.; Kima, Y.H.; Yoob, D.J.; Oha, S.M.; Parkb, S.J. Sensing behaviour of semconducting metal oxides for the detection of organophosphorus compounds. Sens. Actuat. B 1993, 13, 400-403.

36. Bermudez, V.M. Computational study of the adsorption of trichlorophosphate, dimethyl methylphosphonate, and sarin on amorphous $\mathrm{SiO}_{2}$. J Phys. Chem. C 2007, 111, 9314-9323.

37. Segal, S.R.; Suib, S.L. Photoassisted decomposition of dimethyl methylphosphonate over amorphous manganese oxide, catalysts. Chem. Mater. 1999, 11, 1687-1695.

38. Kanan, S.M.; Lu, Z.X.; Tripp, C.P. A comparative study of the adsorption of chloro- and non-chloro-containing organophosphorus compounds on $\mathrm{WO}_{3}$. J. Phys. Chem. B 2002, 106, 9576-9580.

39. Richards, R.; Li, W.; Decker, S.; Davidson, C.; Koper, O.; Zaikovski, V.; Volodin, A.; Rieker, T.; Klabunde, K.J. Consolidation of metal oxide nanocrystals: Reactive pellets with controllable pore structure that represent a new family of porous, inorganic materials. J. Am. Chem. Soc. 2000, 122, 4921-4925. 
40. Yun, K-H, Yun, K-Y, Cha, G-Y, Lee, B. H.; Kim, J-C, Lee, D-D; Huh, J.S. Gas sensing characteristics of $\mathrm{ZnO}$-doped $\mathrm{SnO}_{2}$ sensors for simulants of the chemical agents. Mater. Sci. Forum 2005, 486-487, 9-12.

41. Sauerbrey, G. The use of quartz oscillators for weighing layers and for micro-weighing. Z. Phys. 1959, 155, 206-222.

42. Li, G.; Zheng, J.; Ma, X.; Sun, Y.; Fu, J.; Wu, G. Development of QCM trimethylamine sensor based on water soluble polyaniline. Sensors 2007, 7, 2378-2388.

43. Zheng, J.; Li, G.; Ma, X.; Wang, Y.; Wu, G.; Cheng, Y. Polyaniline-TiO ${ }_{2}$ nano-composite-based trimethylamine QCM sensor and its thermal behavior studies. Sens. Actuat. B 2008, 133, 374-380.

47. Grate, J.W.; Kaganove, S.N.; Bhethanabotla, V.R. Comparisons of polymer/gas partition coefficients calculated from responses of thickness shear mode and surface acoustic wave vapor sensors. Anal. Chem. 1998, 70, 199-203.

45. Martin, S.J.; Frye, G.C.; Senturia, S.D. Dynamics and response of polymer-coated surface-acoustic-wave devices-Effect of viscoelastic properties and film resonance. Anal. Chem. 1994, 66, 2201-2219.

46. Lucklum, R.; Behling, C.; Hauptmann, P. Role of mass accumulation and viscoelastic film properties for the response of acoustic-wave based chemical sensors. Anal. Chem. 1999, 71, 2488-2496.

48. Bodenhofer, K.; Hierlemann, A.; Noetzel, G.; Weimar, U.; Gopel, W. Performances of mass-sensitive devices for gas sensing: Thickness shear mode and surface acoustic wave transducers. Anal. Chem. 1996, 68, 2210-2218.

(C) 2010 by the authors; licensee MDPI, Basel, Switzerland. This article is an open access article distributed under the terms and conditions of the Creative Commons Attribution license (http://creativecommons.org/licenses/by/3.0/). 